\title{
Development of Affordable Effective Vaccines against Whooping Cough for the Developing World
}

\section{Trilochan Mukkur ${ }^{1 *}$ and Peter Richmond ${ }^{2}$}

${ }^{1}$ School of Biomedical Sciences, Curtin Health Innovation Research Institute, Curtin University [Bentley Campus], Perth 6102, Western Australia ${ }^{2}$ School of Paediatrics and Child Health, University of Western Australia, Princess Margaret Hospital for Children, Perth 6340, Western Australia

The rate of mortality in children associated with serious infectious diseases is significantly higher in the developing world and in socioeconomically disadvantaged populations in developed industrialized countries. The reasons underpinning the higher incidence of whooping cough (pertussis) in these populations include overcrowding and poor hygiene, poor coverage with available pertussis containing vaccines and waning immunity following immunisation and infection. Two types of vaccines are currently used for vaccinating infants and children. The whole cell pertussis vaccine, the most used vaccine worldwide, consisting of a killed $B$ pertussis strain, origin of which may differ in different countries, is generally given in combination with chemically or genetically inactivated diphtheria and tetanus toxoids [DTwP or DTPw], with or without combination with Haemophilus influenzae type $\mathrm{b}$ [Hib], hepatitis B or inactivated polio virus vaccine [IPV], depending upon the vaccine manufacturer. This vaccine played a pivotal role in reducing the whooping cough morbidities and mortalities in the 1940's [1]. Because of the perceived seriousness of the side reactions particularly febrile prolonged convulsions or encephalopathy, albeit in insignificant number of vaccinees, has been reported in temporal association with DTwP vaccination [2], the whole cell vaccine was replaced by acellular pertussis vaccine formulations [DTaP] due to better reactogenicity profiles [3]. The aP component in the DTaP formulations consist of chemically or genetically inactivated/detoxified pertussis toxin $[\mathrm{dPT}]$, filamentous haemagglutinin [FHA] and pertactin $[\mathrm{PRN}]$ with or without fimbrial antigens [FIM]. Other components in the DTaP vaccine formulation are the same as mentioned above for the DTwP vaccine with or without combination with Hib, hepatitis $\mathrm{B}$ or inactivated poliovirus vaccine [IPV], depending upon the vaccine manufacturer. The resurgence of pertussis epidemics worldwide and increased proportions of cases in older children and adults, led to the introduction of a adult-formulation acellular pertussis combination vaccine, Tdap, containing reduced pertussis and diphtheria antigen concentrations, for vaccinating adolescents and adults. One recent suggestion to give booster pertussis vaccinations regularly throughout life will not be affordable in developing countries [3], and unlikely to be cost-effective and taken up by socioeconomically disadvantaged groups in developed countries, given the cost of $\mathrm{DTaP}$ at approximately US $\$ 21$ per dose and US \$37 per dose of Tdap. Cost per dose is clearly one reason for the lack of adoption of the DTaP vaccine in resource-poor settings, the production/manufacture of the $\mathrm{wP}$ component of DTwP vaccine being more cost-effective [4]. Due to the potential variability in the performance of different $\mathrm{wP}$ vaccine batches produced as a result of using native $B$ pertussis strains in different countries, it may be better to adopt strains that have been proven to yield high protective efficacy regardless of the origin of the vaccine strain although the regulatory hurdles in different countries are likely to be significant.

Given the high cost of the DTaP and Tdap vaccines, unaffordable by masses in the developing world, efforts to develop alternative affordable vaccines has been confined mainly to DNA and/or live attenuated whooping cough vaccines [5-7], nanoparticle-based pertussis toxoid vaccines having been reported to yield mainly Th2polarised immune responses in the pertussis mouse model [8]. Even though DNA vaccines against whooping cough have yielded conflicting results most likely due to the different routes used for the delivery of vaccine candidates [5-7], better protection is obtained using the primeboost strategy involving boosting with purified recombinant proteins [6] [also Fry S, Daggard G, Chen A and Mukkur T, Unpublished] or granulocyte macrophage colony stimulating factor rendering the cost of the vaccines unaffordable by not only the developing world but also in the industrialized world. However, since the pertussis DNA vaccines induce cell-mediated immune responses, further investigations aimed at developing eukaryotic vectors that can facilitate induction and enhancement of antibody, cell-mediated immune and protective responses when administered as a single dose may yield a cost-effective vaccine product.

An exciting approach, which is not new in concept, is to develop live attenuated, nonreverting, vaccine candidates as has been reported for many other pathogens such as Salmonella species $[9,10]$. The main perceived advantage of live attenuated vaccines is that the infection caused by these vaccine candidates mimics natural infection with potential to induce protective immune responses against most, if not all, the virulence-associated antigens. Development of two live attenuated whooping cough vaccine candidates, a single dose of which has been reported to be sufficient to induce protective immune responses against challenge infection with virulent $B$ pertussis in mouse models. One of these vaccine candidates, aro $Q B$ pertussis, hereafter referred to as aroQBP, is metabolite-deficient and hence avirulent [11], while the second vaccine candidate, BPZE1, has been attenuated by genetic detoxification, and deletion or replacement of select toxins produced by $B$ pertussis [12]. Phase 1 trials on the BPZE1 candidate were carried out in 2011-2012, results of which are awaited for proof of the concept of a live attenuated pertussis vaccine. It will be interesting to evaluate the impact of detoxifications on the immunogenicity of the aroQBP vaccine candidate particularly because of its nonreverting characteristic. Regardless of the prototype live attenuated whooping cough vaccine yielding the best protective immune responses, it is likely to be more cost-effective to produce than the currently marketed DTaP vaccines from a manufacturing perspective. However, it is also important to support intensive efforts to discover Th-1 polarizing adjuvants as a backup, given the potential regulatory constraints in the developed and developing world [13]. In the mean time, however, it is important that infants and children in socially disadvantaged communities in the

${ }^{*}$ Corresponding author: Trilochan Mukkur, School of Biomedical Sciences, Curtin Health Innovation Research Institute, Curtin University [Bentley Campus], Perth 6102, Western Australia, E-mail:T.Mukkur@curtin.edu.au

Received July 03, 2012; Accepted July 06, 2012; Published July 08, 2012

Citation: Mukkur T, Richmond P (2012) Development of Affordable Effective Vaccines against Whooping Cough for the Developing World. J Vaccines Vaccin 3:e107. doi:10.4172/2157-7560.1000e107

Copyright: ( 2012 Mukkur T, et al. This is an open-access article distributed under the terms of the Creative Commons Attribution License, which permits unrestricted use, distribution, and reproduction in any medium, provided the original author and source are credited. 
Citation: Mukkur T, Richmond P (2012) Development of Affordable Effective Vaccines against Whooping Cough for the Developing World. J Vaccines Vaccin 3:e107. doi:10.4172/2157-7560.1000e107

Page 2 of 2

developed world and poor developing nations are provided vaccination against pertussis at affordable rates using the best vaccine available with consideration being given to the application of cocoon vaccination strategy if feasible [14].

\section{References}

1. (2011) National Network for Immunization Information.

2. (1996) Update: Vaccine Side Effects, Adverse Reactions, Contraindications and Precautions Recommendations of the Advisory Committee on Immunization Practices (ACIP). MMWR Recomm Rep 45: 1-35.

3. Poolman JT, Hallander H, Halperin SA [2011] Pertussis vaccines: where to now? Expert Rev Vaccines 10: 1497-1500.

4. Julie Milstien, Sarah Glass, Amie Batson, Michel Greco, Jacques Berger (2002) Divergence of vaccine product lines in industrialized and developing countries.

5. Kamachi K, Konda T, Arakawa Y (2003) DNA vaccine encoding pertussis toxin $\mathrm{S} 1$ subunit induces protection against Bordetella pertussis in mice. Vaccine 21: 4609-4615.

6. Li QT, Zhu YZ, Chu JY, Dong K, He P, et al. (2006) Granulocyte-macrophage colony-stimulating factor DNA prime-protein boost strategy to enhance efficacy of a recombinant pertussis DNA vaccine. Acta Pharmacol Sin 27: 1487-1494.

7. Fry SR, Chen AY, Daggard G, Mukkur TK (2008) Parenteral immunization of mice with a genetically inactivated pertussis toxin DNA vaccine induces cellmediated immunity and protection. J Med Microbiol 57: 28-35.
8. Conway MA, Madrigal-Estebas L, McClean S, Brayden DJ, Mills KH (2001) Protection against Bordetella pertussis infection following parenteral or ora immunization with antigens entrapped in biodegradable particles: effect of formulation and route of immunization on induction of Th1 and Th2 cells. Vaccine 19: 1940-1950.

9. Hoiseth SK, Stocker BA (1981) Aromatic-dependent Salmonella typhimurium are non-virulent and are effective live vaccines. Nature 291: 238-239.

10. Curtiss R 3rd, Kelly SM (1987) Salmonella typhimurium deletion mutants lacking adenylate cyclase and cyclic AMP receptor protein are avirulent and immunogenic. Infect Immun 55: 3035-3043.

11. Cornford-Nairns R, Daggard G, Mukkur T (2012) Construction and Preliminary Immunobiological Characterization of a Novel, Non-Reverting, Intranasal Live Attenuated Whooping Cough Vaccine Candidate. J Microbiol Biotechnol 22 856-865.

12. Mielcarek N, Debrie AS, Raze D, Bertout J, Rouanet C, et al. (2006) Live attenuated $B$. pertussis as a single-dose nasal vaccine against whoopingcough. PLoS Pathog 2: e65.

13. Milstein J, Belgharbi L (2004) Regulatory pathways for vaccines for developing countries. Bull World Health Organ 82: 128-133.

14. Coudeville L, Van Rie A, Getsios D, Caro JJ, Crépey P, et al. (2009) Adult Vaccination Strategies for the Control of Pertussis in the United States: An economic evaluation including the dynamic population effects. PLoS ONE 4 e6284. 